\title{
Another test of the Premack principle
}

\author{
ROBERT G. HARRISON \\ Anclote Manor, Tarpon Springs, Florida 33589 \\ and \\ ROBERT W. SCHAEFFER \\ Auburn University, Auburn, Alabama 36830
}

\begin{abstract}
A failure to support Premack's assertion that a rate reduction in the reinforcing response of a reinforcement contingency, relative to the precontingency baseline rate of that response, is necessary for the occurrence of reinforcement, is reported. The implications of this finding for reinforcement theory are discussed, and it is proposed that reinforcement contingencies have potential importance for evaluating animal intelligence.
\end{abstract}

Premack (1965) has suggested that the reinforcement relationship includes, in addition to the commonly recognized temporal contiguity of a less probable instrumental response (L) and a more probable reinforcing response $(\mathrm{H})$, three less commonly recognized experimenter-imposed conditions: (1) an instrumental response requirement, (2) a reduction in the availability of $H$, relative to the case where $\mathrm{H}$ is freely available to the subject, and (3) a circumscribing of the distribution of $\mathrm{H}$, relative to the case where the subject is unhampered by the constraints of experimenter-programmed access to $\mathrm{H}$ and is free to distribute its $\mathrm{H}$ responding in any chosen fashion. Premack (1965) acknowledged the importance of the if $L$, then $H$ temporal relationship in reinforcement procedures but has proposed that a reduced availability of $H$, relative to its baseline occurrence, is the crucial factor in conditioning. An adequate test of this specific proposal is not possible, however, so long as both the distribution and availability of $\mathrm{H}$ are manipulated at the same time, as they always are in the traditional reinforcement paradigm where a limited opportunity to engage in $\mathrm{H}$ is contingent upon the occurrence of $\mathrm{L}$. It is unlikely that any contingency can be arranged that does not put some restriction on the occurrence of $\mathrm{H}$, since any reinforcement contingency requires a sequential if $L$, then $H$ relationship. There is a method, however, whereby both the extent of the experimenter's circumscription of $\mathrm{H}$ responding and any experimenter-programmed reduction in $\mathrm{H}$ may be minimized.

In the present experiment, when an animal completed an instrumental running requirement in an activity wheel, it gained unlimited access to a sucrose solution for the remainder of the session. Both the

This research was supported in part by Public Health Research Grants MH-08775 and MH-12025 from the National Institute of Mental Health, R. W. Schaeffer, principal investigator. length of the experimental session and the instrumental response requirement employed were such that neither session length nor the response requirement initially competed with the amount of time available to the animal for engaging in the reinforcing response. The present experiment, therefore, provided a straightforward test of Premack's contention that a reduction of the availability of $H$ is a necessary condition for the occurrence of reinforcement.

\section{METHOD}

Two naive adult female albino Dublin SDD/DR rats were maintained on an 11.5-h water-deprivation schedule with ad-lib food. Two instrumented activity wheels, that have been described in detail elsewhere (Schaeffer, 1966), were employed. Standard electromechanical circuitry provided programmed reinforcement delivery (access to the drinking tube) and recorded lick contacts and $180^{\circ}$ wheel revolutions. Responses and contingency manipulations were recorded by Esterline-Angus analog-digital operations recorders. Fluid ingestion was recorded directly from 100-ml graduated cylinders.

The five-phase (ABACA) experiment utilized concurrent baselines (phase $A$ ) in which running $(L)$ and licking $(H)$ were freely and concurrently available, alternated with FR conditions (phases $B$ and $C)$ in which drinking was contingent upon running. All sessions were $0.5 \mathrm{~h}$ in length and spaced $11.5 \mathrm{~h}$ apart. The stability criterion employed in all but the second FR condition (phase C) of the experiment required that mean responding for the last four sessions minus mean responding of the preceding four sessions divided by mean responding of the last eight sessions not exceed $20 \%$ for each animal (Schoenfeld. Cummings, \& Hearst, 1956). In the second FR condition (phase C), the animals were run for a total of 10 consecutive sessions at each FR value tested.

In the first FR condition (phase B), the animals received contingency training on an FR 20 run-to-lick schedule until stable responding was obtained. In this phase, when the animals completed the instrumental response requirement of 20 wheel turns $\left(180^{\circ}\right)$, the drinking tube was made available for a contingent time period of $20 \mathrm{sec}$.

The second FR condition (phase C) consisted of increasing FR requirements programmed for each animal. Completion of the FR response requirement by each animal made reinforcement available for the entire remainder of the 0.5 -h session. Each specific FR value in this phase was retained for a total of 10 sessions; the animals 
EXPERIMENTAL CONDITIONS

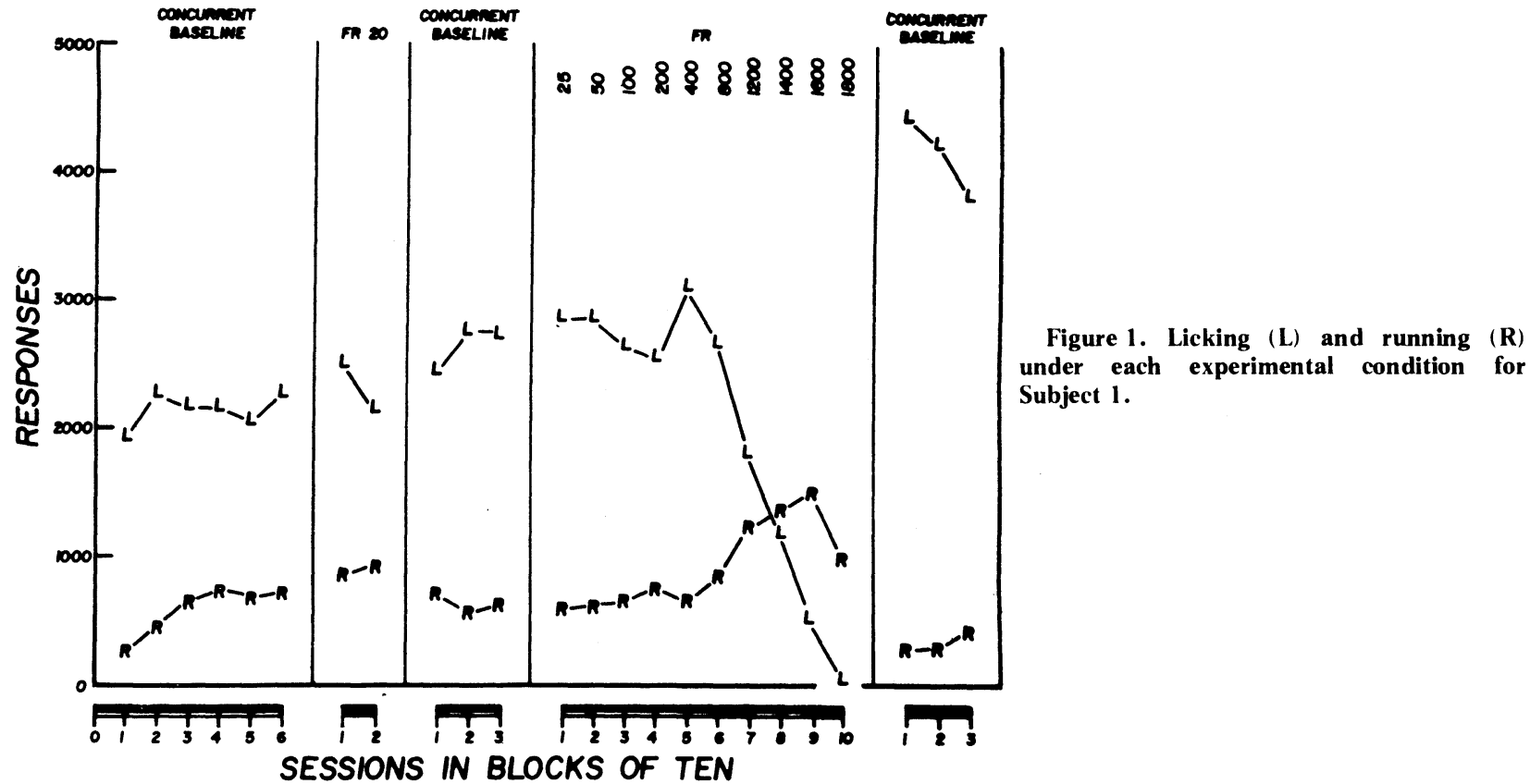

were then shifted to the next higher FR value. Phase $C$ of the experiment was terminated for each animal when it did not complete the $F R$ requirement (i.e., earned no reinforcement) for nine consecutive sessions.

Following phase $C$. each animal was shifted to the third concurrent baseline condition. This third baseline condition was identical to the first two concurrent baseline conditions in all respects.

\section{RESULTS AND DISCUSSION}

Figures 1 and 2 summarize the data obtained for the animals. Each data point shown in the figures represents mean responding across 10 consecutive sessions. All data obtained in the experiment were evaluated by analysis of variance techniques suitable

\section{EXPERIMENTAL CONDITIONS}

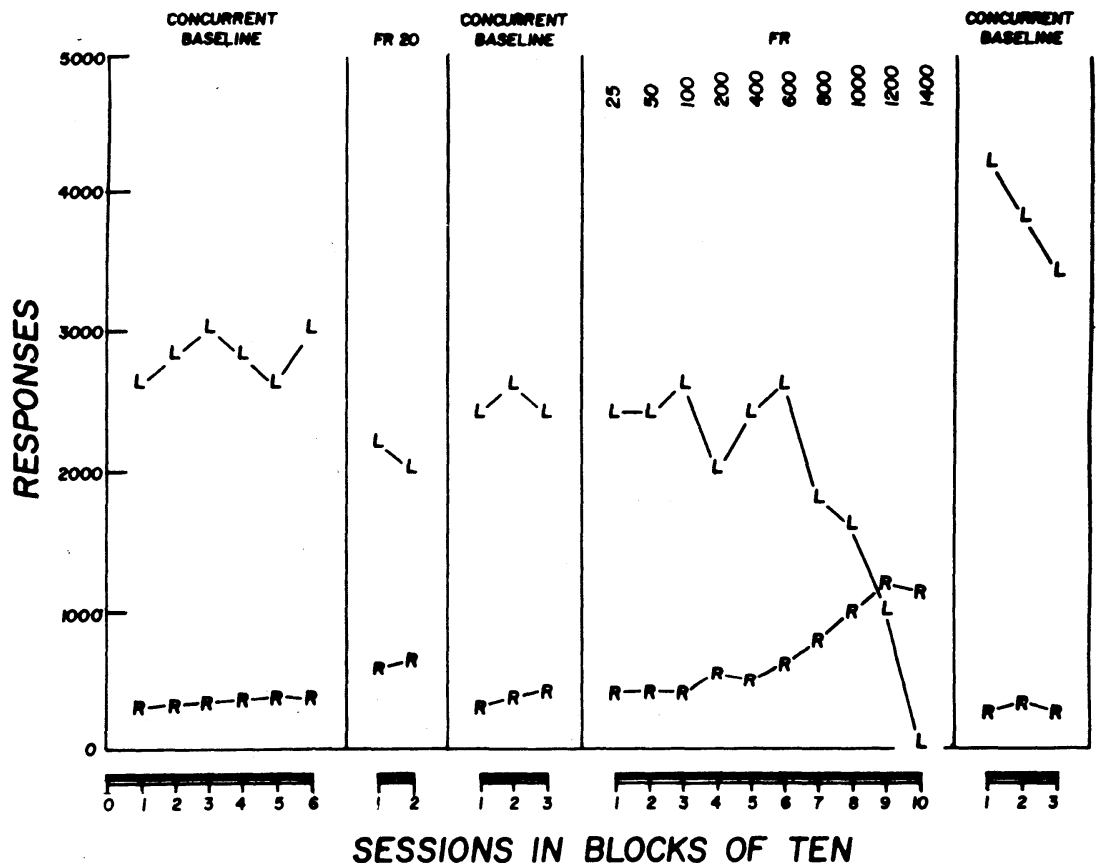

Figure 2. Licking (L) and running ( $R$ ) under each experimental condition for Subject 2. 
for repeated, within-subject measures. Significant ANOVA differences were evaluated by Duncan's multiple range test. Statistically significant differences were inferred from tests in which the value of $\mathrm{p}<.01$.

For both animals in phase $\mathrm{C}$ of the experiment, a statistically significant increase in instrumental running (L) was obtained before a statistically significant decrement in the reinforcing drinking response (H) was obtained. For Subject 1, the increment in running was found at FR 800 , whereas a decrement in drinking did not occur until FR 1200. For Subject 2, the increment in running was obtained at FR 600, whereas a reduction in drinking was not obtained until FR 1000. If a reduction in $H$ is the crucial factor in conditioning, as Premack (1965) has proposed, then both animals should have demonstrated a reduction in contingent drinking (relative to the immediately preceding concurrent baseline in which running and drinking were both freely available) before an increment in the instrumental running response was obtained. Clearly, a reduction in $\mathrm{H}$ was not, for the $\mathrm{FR}$ paradigm employed in phase $\mathrm{C}$ of the experiment, a necessary condition for the occurrence of reinforcement.

Since the FR paradigm employed in phase $\mathrm{C}$ of this experiment represented a marked procedural departure from the normal FR procedure which was employed in phase B, a direct comparison of the performance of the animals under the standard FR 20 condition in phase B with the atypical FR paradigm used in phase $C$ was made. Comparisons of running in the FR 20 and in the first concurrent baseline (phase $A$ ) revealed substantial increments in running under the FR condition for both animals $(p<.01)$. It is of primary interest to note that neither of the animals showed a decrement $(p>.05)$ in licking, when licking in the FR 20 (phase B) was compared with licking in the concurrent baseline. Clearly, a reduction in $\mathrm{H}$ was not a necessary condition for the occurrence of reinforcement for either of the FR paradigms employed here.

In a previous study (Harrison $\&$ Schaeffer, 1975), it was demonstrated that mere temporal contiguity of a less probable response and a more probable response, with the occurrence of the less probable response preceding the more probable response, was not a sufficient condition for reinforcement to occur. Delay of reinforcement studies (e.g., Frazier, 1968) also indicate that temporal contiguity is not a necessary condition for reinforcement to occur. Furthermore, an instrumental response requirement is neither a necessary nor a sufficient condition for reinforcement, as is attested both by the "superstitious" behavior generated by response-independent reinforcement schedules and by the failure of conditioning to occur for low probability responses. Finally, the results of the present study and another study (Harrison, 1970) suggest that, contrary to Premack's proposal, a reduction in the availability of the reinforcing response is neither necessary nor sufficient for reinforcement to occur.

If none of the foregoing properties of the reinforcement relation are either necessary or sufficient conditions for reinforcement to occur, what then are the essential components of the reinforcement relationship which do result in reinforcement? As we indicated above, it is unlikely that any contingency can be arranged that does not in some way restrict the availability of the reinforcing response or potentially alter the way in which the subject distributes its responding, relative to the manner in which the subject responds when the reinforcing response and the reinforcer are freely available. When stripped of all other possible nonessential defining properties, however, it would appear that the only essential characteristic that defines a reinforcement contingency is the inference of the contingency itself, in which the availability of one response is either (a) actually made contingent upon some other response or (b) responded to by the subject as though the response were contingent upon some other response. Operationally, the preceding statement provides a parsimonious description of the reinforcement procedures utilized in all operant conditioning studies, including those that have been used to demonstrate adventitious reinforcement effects.

Experimentally, it is quite reasonable to ask why reinforcement contingencies should be effective in maintaining behavior. Premack (1965) has suggested that the reinforcement contingency is effective only because access by the organism to the experimenterprogrammed reinforcing response is a condition which the animal prefers. Stated in its most simple form, the Premack principle avers that effective reinforcement involves no more than the arrangement of a contingency between more probable and less probable responses in an organism's behavioral repertoire.

When subjected to further scrutiny, however, the application of reinforcement contingencies involves two other identifiable aspects that deserve more attention from reinforcement theorists. These two aspects are (a) the arrangement of the contingency by the experimenter, with the particular reinforcement contingency typically being more dependent upon the experiential history and mechanical ingenuity of the experimenter than dependent upon the organism's full response capabilities, and (b) the ability of the species being tested to attend and respond to the fact that a contingency has been imposed upon it. Stated somewhat differently, unless one assumes complete automaticity of the reinforcement procedure, the use of the reinforcement contingency assumes the ability of the animal to ferret out the contingency in which it 
has been imprisoned by the experimenter. Clearly, even when a specitic contingency has not been explicitly programmed by the experimenter, predictable behavioral consequences of accidental environment-response correlations (i.e., superstitious behavior) suggest implicit recognition of the correlation by the organism. It is interesting to speculate that the failure to conduct systematic between-species comparisons of the abilities of organisms to accurately detect cause-effect relationship (or conversely, to discriminate accidental and inconsequential environment-response correlations) may have impeded comprehensive understanding of the process of reinforcement as an intraorganismic event.

Given that an organism has the capacity to correctly respond to a cause-effect relationship to which it is exposed. the organism has available to it the separate alternative as to whether it will or will not respond. Functionally, this alternative has been acknowledged in the distinction between learning and performance. Traditionally, experimenters have attempted to circumvent the potential problem of nonperformance in the presence of learning by reducing the opportunity of the organism to engage in the reinforcing response outside of the contingency that is programmed by the experimenter. The capacity of an organism to recognize and resolve experimental situations which the experimenter presents was identified early by Thorndike (1911) as animal intelligence. If intelligence may be defined, in part, in terms of the ability of an organism to recognize and to respond or not to respond to reinforcement contingencies in which they are imprisoned, it is clear that the entire spectrum of reinforcement procedures suggested by the Premack principle warrants considerable further attention. It is also clear that investigations involving various species must be conducted under conditions where the probability of the organism's responding to the reinforcement contingency must be accurately predicted and systematically manipulated. Given the data that can be provided by such experiments, both the process and the procedures of reinforcement eventually may become more fully understood.

\section{REFERENCES}

Frazier, J. R. The effects of constant delay of reinforcement on extinction responding. Unpublished doctoral disseration. Florida State University. 1968.

Harrison, R. G. Investigations of Premackian reinforcement theon. Unpublished doctoral dissertation. Florida State University. 1970.

Harrison. R. G.. \& Schaeffer, R. W. Temporal contiguity: Is it a sufficient condition for reinforcement? Bulletin of the Psychonomic Society. 1975, 5. 230-232.

Premack. D. Reinforcement theory. In D. Levine (Ed.), Nehraska Symposium on Motivation. 1965. Lincoln. Nebr: University of Nebraska Press. 1965.

Schaeffer, R. W. A new device for programming contingencies between drinking. running. and lever-pressing. Journal of the Experimental Analysis of Behavior. 1966. 9. 529-533.

Schoenfei.d. W. N.. Cumming, W. W.. \& Hearst, E. On the classification of reinforcement schedules. Proceedings of the National Academy of Science. 1956, 42. 563-570.

Thorndike, E. L. Animal intelligence. New York: Hafner, 1911.

(Received for publication July 31, 1975.) 